\title{
DETERMINACIÓN E INCIDENCIA DE LA DISPOSICIÓN A PAGAR EN ESQUEMAS DE PAGOS POR SERVICIOS AMBIENTALES HÍDRICOS: ESTUDIO DE CASO EN LAS CAPITALES DE LAS PROVINCIAS DE CHACHAPOYAS, RODRÍGUEZ DE MENDOZA Y UTCUBAMBA
}

\author{
Wagner GUZMÁN CASTILLO', Erick Stevinsonn ARELLANOS CARRIÓN² , Segundo Grimaldo \\ CHAVEZ QUINTANA ${ }^{3}$ \\ 1 Instituto de Investigaciones de la Amazonía Peruana (IIAP) - Filial Amazonas. Jirón Junín Nº625 - Chachapoyas. E-mail: \\ wguzman@iiap.org.pe \\ 2 Ingeniero Agroindustrial. Chachapoyas. E-mail: erick_ac7@hotmail.com \\ 3 Becario del Instituto de Investigaciones de la Amazonía Peruana (IIAP) - Universidad Nacional Toribio Rodríguez del \\ Mendoza.E-mail: shegho@hotmail.com
}

\begin{abstract}
RESUMEN
Chachapoyas, San Nicolás y Bagua Grande son tres de las cuatro ciudades más importantes económica y demográficamente de la región Amazonas, ubicadas en zona andina, ceja de selva y selva baja, respectivamente. El deterioro de las fuentes de agua, debido principalmente a las actividades no acordes a la capacidad de uso del suelo, ha ocasionado problemas particulares en la disponibilidad del recurso. Chachapoyas obtiene agua del Área de Conservación Privada Tilacancha donde abunda este recurso, San Nicolás dispone de agua parcialmente durante el día del Área de Conservación Ambiental Municipal Huamanpata, y Bagua Grande, que presenta una seria escasez de este recurso lo obtiene del Cerro Shipago (actualmente en proyecto de área para la conservación). En el presente estudio se aplicó el Método de Valoración Contingente (MVC) a fin de estimar la Disponibilidad a Pagar (DAP) de los usuarios dirigida a acciones de conservación de la fuentes agua; para ello, se aplicaron encuestas a jefes de familia en el año 2012. Las encuestas definitivas tuvieron el formato dicotómico doble con seguimiento o "double bounded", cuyos montos ofertados de DAP se eligieron de la encuesta piloto. Los métodos econométricos estimaron montos en Chachapoyas de 2.24 S/mes; San Nicolás, $2.97 \mathrm{~S} /$ mes y Bagua Grande, $1.95 \mathrm{~S} / \mathrm{mes}$. Las variables más significativas e incidentes en la DAP fueron: monto hipotético de pago, ingreso económico familiar, nivel educativo, relación bosque-provisión de agua y calidad de agua. Finalmente, los montos anuales estimados para el total de usuarios pueden constituir fondos semilla para implementar futuros esquemas de pagos por servicios ambientales.

PALABRAS CLAVE: Valoración Contingente, Disposición a Pagar, Double Bounded, Pagos por Servicios Ambientales.

\section{INCIDENCE AND DETERMINATION OF THE DISPOSITION TO PAY EN SCENARIOS OF PAYMENTS FOR NATURAL WATER SERVICES: CASE STUDY IN THE CAPITALS OF THE PROVINCES OF CHACHAPOYAS, RODRIGUEZ DE MENDOZA AND UTCUBAMBA \\ ABSTRACT}

Chachapoyas, San Nicolas and Bagua Grande are three of the four economically and demographically most important cities in the Amazon region, located in the Andean region, Andeans piedmont and jungle lowlands, respectively. The deterioration of water sources, mainly due to the activities not in accordance with the land use capacity, has caused particular problems in resource availability. Chachapoyas obtains water from the Tilacancha Private Conservation Area where this resource abounds; San Nicolas has water partially during the day from the Huamanpata Municipal Environmental Conservation Area and Bagua Grande, with a serious scarcity of this resource, obtains water from the Shipago Mountain (now days in draft to be a conservation area). In the present study the Contingent Valuation Method (CVM) was used to estimate the willingness to pay (WTP) of users actions aimed at conserving water sources, for this, surveys were administered to heads of households in the year 2012. The final survey had the double dichotomous monitoring or "double bounded" whose amounts offered were chosen WTP as pilot survey. Econometric methods in Chachapoyas estimated amounts of $2.24 \mathrm{~S} /$ month; in San Nicolas $2.97 \mathrm{~S} /$ month and in Bagua Grande $1.95 \mathrm{~S} /$ month. The most significant variables and incidents in WTP were: hypothetical payment amount, family income, education level, relationship between forests and water supply and water quality. Finally, the annual estimations for the total number of users can provide seed funding to implement future payment schemes for environmental services.

KEYWORDS: Contingent Valuation, Willingness to Pay, Double Bounded, Payments for Environmental Services. 


\section{INTRODUCCIÓN}

Durante mucho tiempo los bienes y servicios ambientales han sido vistos como recursos infinitos, sin embargo, con el crecimiento exponencial de la población, nos vamos dando cuenta que estos son cada vez más escasos y las fuentes de los mismos se encuentra en graves riesgos; en tal sentido, se hace necesario generar herramientas que garanticen su continuidad y optimicen su aprovechamiento.

El Perú, en relación a la distribución geográfica de su población, es un país de escasos recursos hídricos con condiciones de extrema aridez, que otorgan al agua un alto valor económico y generan conflictos de interés y competencia entre diferentes áreas geográficas y tipos de usuarios (Loyola, 2007). A nivel nacional se ha venido trabajando para conservar las fuentes de recursos naturales, de tal manera que el agua que abastece a las principales ciudades provienen de Áreas Naturales Protegidas (ANP) y otras se encuentran en proceso de catalogarse como tales.

El agua suministrada a la ciudad de Chachapoyas, proviene de la Área de Conservación Privada Tilacancha (Reconocida mediante RM No 118-2010MINAM sobre los predios de las Comunidades Campesinas de San Isidro del Maino y Levanto); San Nicolás consume el agua proveniente del Área de Conservación Ambiental Municipal Huamanpata (Creada por la municipalidad provincial de Rodríguez de Mendoza el año 2006) y por otro lado la ciudad de Bagua Grande se abastece con agua proveniente del Cerro Shipago, cerro que integra el corredor montañoso Vilaya Condorpuna Shipago (VICONSHI), ubicada en las provincias de Utcubamba y Luya (Se encuentra catalogado como Sitio Prioritario para la conservación según el Sistema de Conservación Regional Amazonas SICRE).

Sin embargo, estas zonas proveedoras del recurso hídrico se encuentran seriamente amenazadas por actividades productivas de las poblaciones que se ubican en su ámbito geográfico. La ACP Tilacancha pertenece a las Comunidades Campesinas de Mayno y Levanto; en la ACA Huamanpata, encontramos posesionarios de parte de las tierras y el resto se encuentra en peligro de ser invadida por extractores de madera principalmente. En el cerro Shipago encontramos asentamientos humanos dedicados principalmente a la producción agropecuaria no sostenible, que afectan directamente a los recursos y fuentes de agua.

En los tres casos se ve la necesidad de implementar sistemas que permitan poner fin al aprovechamiento insostenible y que además garanticen el abastecimiento adecuado del recurso hídrico a las poblaciones. Los esquemas de PSA en nuestro país aún no cuentan con un marco normativo legal, sin embargo existen esfuerzos por cambiar esta situación en el corto plazo. En tal sentido el gobierno en todos sus niveles y la población beneficiaria están involucrados en la gestión del recurso a fin de garantizar su aprovechamiento sostenible. La Valoración Económica (VE) de la fuente hídrica para las tres ciudades busca capturar las preferencias sociales de preservar ciertos niveles de bienestar generados por los beneficios de su conservación y su uso sustentable.

Los esquemas de PSA son instrumentos que permiten a los agentes afectados positivamente por un determinado manejo ambiental, remuneren a quienes lo llevan a cabo por hacerlo en el entendido de que, de otra forma no lo harían (Azqueta, 2007). Los esquemas de PSA constituyen, por tanto, una herramienta que puede garantizar la provisión a futuro del servicio ambiental hídrico. Pagiola et al. (2005), enlista los pocos pero ejemplares avances regionales en la implementación de PSA: Costa Rica tiene un sistema nacional de PSA, México un sistema nacional llamado Pago por Servicios Ambientales Hidrológicos, la ciudad de Quito tiene un fondo de agua donde contribuyen las compañías eléctricas para proteger las cabeceras de cuenca de donde obtienen el agua y Colombia, donde grupos regantes pagan por la conservación de las cuencas. El común de estos ejemplos es que son experiencias locales que no generan divisas para los países pero que ayudan a la conservación de los recursos naturales.

Es sorprendente que, en Perú, si bien aún no hay marco legal para implementar esquemas de PSA, existe la experiencia documentada sobre la implementación de un esquema de PSA en la Ciudad de Moyobamba (MINAM, 2010), sin embargo, ésta no se ha constituido sobre bases coyunturales sólidas lo que ha conllevado que no funcione como tal, además, no está claro cuáles fueron los mecanismos para esquivar la normativa vigente. En este sentido el presente estudio tiene por objetivo determinar y evaluar la DAP en condiciones sociales, económicas y ambientales diferentes como base para proponer mecanismos de PSA en las capitales de las provincias de Chachapoyas, Rodríguez de Mendoza y Utcubamba.

\section{MATERIAL Y MÉTODOS}

\section{DESCRIPCIÓN DE LOS LUGARES DE ESTUDIO}

Chachapoyas

La ciudad de Chachapoyas es la capital de la Región Amazonas, a una altitud de 2300 m.s.n.m., cuenta con 23202 habitantes (INEI, 2007), se abastece de recurso hídrico de la Cuenca del río Tilacancha, 
ubicadas en la ACP del mismo nombre, en las Comunidades Campesinas (CC.CC) de San Isidro del Maino y Levanto. La mencionada cuenca, ubicada en zona andina a unos 3600 m.s.n.m., está conformada por extensos pajonales cubiertos parcialmente por arbustos. Las principales actividades en las comunidades son la ganadería vacuna y la agricultura (tubérculos, cereales y hortalizas). Con el deseo de que la cuenca pueda garantizar a futuro el abastecimiento de agua a todas las familias que dependen de ella, se ha conformado un grupo técnico en la ciudad de Chachapoyas con autoridades locales, instituciones ambientales y ONGs cuyo primer logro es la ACP Tilacancha.

\section{San Nicolás - Mariscal Benavides}

La ciudad de San Nicolás, capital de la Provincia de Rodríguez de Mendoza, y Mariscal Benavidez se abastecen de la misma fuente de agua, por lo que para efectos prácticos en el presente estudio se nombran a ambas, como San Nicolás, con todas sus características socio-demográficas. San Nicolás posee 2409 habitantes (INEI, 2007), se abastece de agua de las quebradas denominadas Inguilpata y Shaylla, las cuales provienen de la Área de Conservación Ambiental Municipal Huamanpata. Durante las últimas décadas el avance de la ganadería ha conllevado a que los bosques inmediatos a Huamanpata se hayan reducido peligrosamente, lo cual ha repercutido en la calidad y cantidad del agua, generándose un malestar social.

\section{Bagua Grande}

Es la ciudad más poblada de la región Amazonas. Cuenta con 29507 habitantes (INEI, 2007), se abastecen de agua proveniente del Cerro Shipago, el cual se encuentra muy deteriorado debido al avance de la agricultura y ganadería. Se calcula que en la provincia existe un $85 \%$ de deforestación (PEJSB, 2009), razones de imperiosa necesidad para las autoridades locales de emprender gestiones dirigidas a adoptar esquemas de PSA para mitigar el problema de escasez, que se evidencia en 3 horas de suministro de agua cada 2 días.

\section{HIPÓTESIS DE TRABAJO}

Es posible determinar valores de disposición de pago DAP ante situaciones hipotéticas de mejora que varían de acuerdo a condiciones sociales, económicas y ambientales distintas, y permiten orientar la implementación de esquemas de pago por servicios ambientales hídricos.

\section{Determinación del tamaño de muestra}

La población de estudio fueron todos los hogares asentados en las tres ciudades. Para efectos de cálculo se empleó la relación estadística de muestreo probabilístico. (Tabla 1)

$$
n=\frac{Z^{2} q p N}{E^{2} N-1+Z^{2} q p}
$$

Dónde:

$\mathrm{N}$ = población (número de familias)

$\mathrm{n}=$ tamaño de la muestra probabilística

$Z=1.96$ (según tabla estadística al $95 \%$ de confianza)

$\mathrm{P}=0.95$ (nivel de aceptación)

$\mathrm{Q}=0.05$ (nivel de fracaso)

$\mathrm{E}=0.05$ (error de estimación)

\section{Diseño y ejecución de encuestas piloto}

Se elaboró una encuesta piloto de carácter exploratorio, para averiguar aspectos generales de la población. Esta encuesta sirvió para encontrar y establecer los rangos a tener en cuenta en la encuesta definitiva. La encuesta constó de tres partes: la primera con preguntas relacionadas al estado actual del servicio de agua potable, la segunda sobre preguntas en el escenario contingente y por último, preguntas socioeconómicas. Se aplicaron 100 encuestas piloto por ciudad y el entrevistado fue el jefe de cada hogar seleccionado al azar.

\section{Diseño y ejecución de encuestas definitivas}

Con la información obtenida y sistematizada de la encuesta piloto se elaboró la encuesta definitiva con las mismas partes y bajo el formato dicotómico doble con seguimiento o double bounded (Figura 1), que contiene el vector de pagos establecido. Las encuestas fueron aplicadas entre los meses de noviembre 2012 a enero 2013 en cada una de las ciudades a los jefes de hogar, obedeciendo a una distribución sistemática-aleatoria, tal que las viviendas comprendidas por manzana tengan la misma probabilidad de ser elegidas. Se ordenó, codificó y jerarquizó la data de encuestas definitivas en forma numérica en hojas de cálculo Excel. Se asignaron rangos a las variables cualitativas y las variables cuantitativas conservaron los valores obtenidos.

\section{ANÁLISIS Y MODELAMIENTO ESTADÍSTICO}

Los datos se trabajaron estadísticamente utilizando el software Nlogit vs 3.0 y Stata vs 12.0, estimando modelos paramétricos logit, cuyos coeficientes son estimados por Máxima Verosimilitud. Los resultados definieron el modelo óptimo, considerando la significancia estadística de las variables y la consistencia teórica de los signos de dichas variables. 


\section{ESPECIFICACIÓN ECONOMÉTRICA DE LOS MODELOS LOGIT}

Chachapoyas:

$\operatorname{Prob}(\mathrm{Si})=\Delta \mathrm{U}=\alpha_{0}+\alpha_{1}{ }^{*} \log (\mathrm{M})+\alpha_{2}{ }^{*}$ ING $+\alpha_{3}{ }^{*}$ EDU $+\alpha_{4}{ }^{*}$ REL

San Nicolás:

$\operatorname{Prob}(\mathrm{Si})=\Delta \mathrm{U}=\alpha_{0}+\alpha_{1}{ }^{*} \log (\mathrm{M})+\alpha_{2}{ }^{*} \operatorname{NG}+\alpha_{3}{ }^{*} \mathrm{EDU}+\alpha_{4}{ }^{*} \mathrm{CS}$

Bagua Grande:

$\operatorname{Prob}(\mathrm{Si})=\Delta \mathrm{U}=\alpha_{0}+\alpha_{1}{ }^{*} \log (\mathrm{M})+\alpha_{2}{ }^{*} \operatorname{NG}+\alpha_{3}{ }^{*} \mathrm{EDU}+\alpha_{4}{ }^{*} \mathrm{CA}$

Dónde:

$\Delta \mathrm{U}=$ Variación de la función de utilidad

$\mathrm{DAP}=$ Disponibilidad a Pagar $(\mathrm{Si}=1, \mathrm{No}=0)$

$\alpha_{0} \quad=$ Intercepto

$\alpha_{i} \quad=$ Coeficientes de las variables

$\mathrm{M}=$ Monto Hipotético a Pagar

$\mathrm{ING}=$ Ingreso

$\mathrm{EDU}=$ Nivel Educativo

$\mathrm{REL}=$ Relación Agua-Bosque $(\mathrm{Si}=1, \mathrm{No}=0)$

$\mathrm{CS}=$ Calidad del servicio de la empresa proveedora de agua

$\mathrm{CA}=$ Calidad del agua

\section{RESULTADOS}

ESTADÍSTICA DESCRIPTIVA: RELACIÓN AGUAVEGETACIÓN, PERCEPCIÓN SOBRE CANTIDAD DE AGUA Y VEHÍCULO DE PAGO DEL MONTO A PAGAR

Las poblaciones de las tres ciudades creen que hay una relación entre el agua y la vegetación, reflejado en un $100 \%, 89 \%$ y $82 \%$ en orden descendente para San Nicolás, Bagua Grande y Chachapoyas respectivamente. La cantidad de agua es insuficiente para Chachapoyas, San Nicolás y Bagua Grande (en $33 \%, 51 \%$ y $88 \%$ respectivamente); lo que indica que la gravedad del problema aumenta conforme el orden indicado. Un 79\%, 52\% y 98\% de la población de Chachapoyas, San Nicolás y Bagua Grande respectivamente creen que el vehículo de pago en un esquema de PSA debería ser el recibo de agua. Para San Nicolás observamos que un $46 \%$ cree que se podría realizar el pago de forma directa a la municipalidad, debido probablemente a que en la actualidad en esta ciudad no se está pagando por el servicio (Figura 2).

ANÁLISIS DE PROBABILIDADES: DAP VS MONTO A PAGAR, DAP VS INGRESO Y DAP VS NIVEL EDUCATIVO

El las tres ciudades encontramos, tal como señala la bibliografía, la DAP es inversamente proporcional al monto ofrecido y directamente proporcional al ingreso y educación respectivamente (Figura 3,4 y $5)$.

Las respuestas "No" al primer y segundo monto ofertado son valores altos (Tabla 2), sin embargo, a estas personas se hizo una pregunta adicional sobre una parte del monto que paga por el servicio sea destinado a las acciones de conservación y aprovechamiento sostenible del recurso, observamos que la negación rotunda a cooperar disminuye a $30.85 \%, 14.29 \%$ y $20.22 \%$ para las ciudades de Chachapoyas, San Nicolás y Bagua Grande respectivamente (Tabla 3). Observamos que estos valores presentan una relación inversa a los problemas de abastecimiento en las tres ciudades. El porcentaje de negación (Tabla 3) está en función al total de encuestas aplicadas.

Conforme a la literatura, se corroboran signos adecuados de la Regresión Logit (Tabla 4), para las variables M, ING, EDU, REL, CS y CA. Sumado a ello, según la significancia estadística de las variables se tiene la seguridad de calcular la DAP con el modelo adecuado.

Los resultados (Tabla 5), indican que San Nicolás presenta la más alta DAP (S/mes) y en último orden, Bagua Grande. Sin embargo, debido a que San Nicolás y Bagua Grande tiene escasez de agua (VAG), tienen por tanto, Mayor DAP por metro cúbico $\left(\mathrm{m}^{3} / \mathrm{mes}\right)$ de consumo. Finalmente, para efectos de comparación se recomienda analizar el primer valor, que representa la DAP de consumo al mes por familia. 


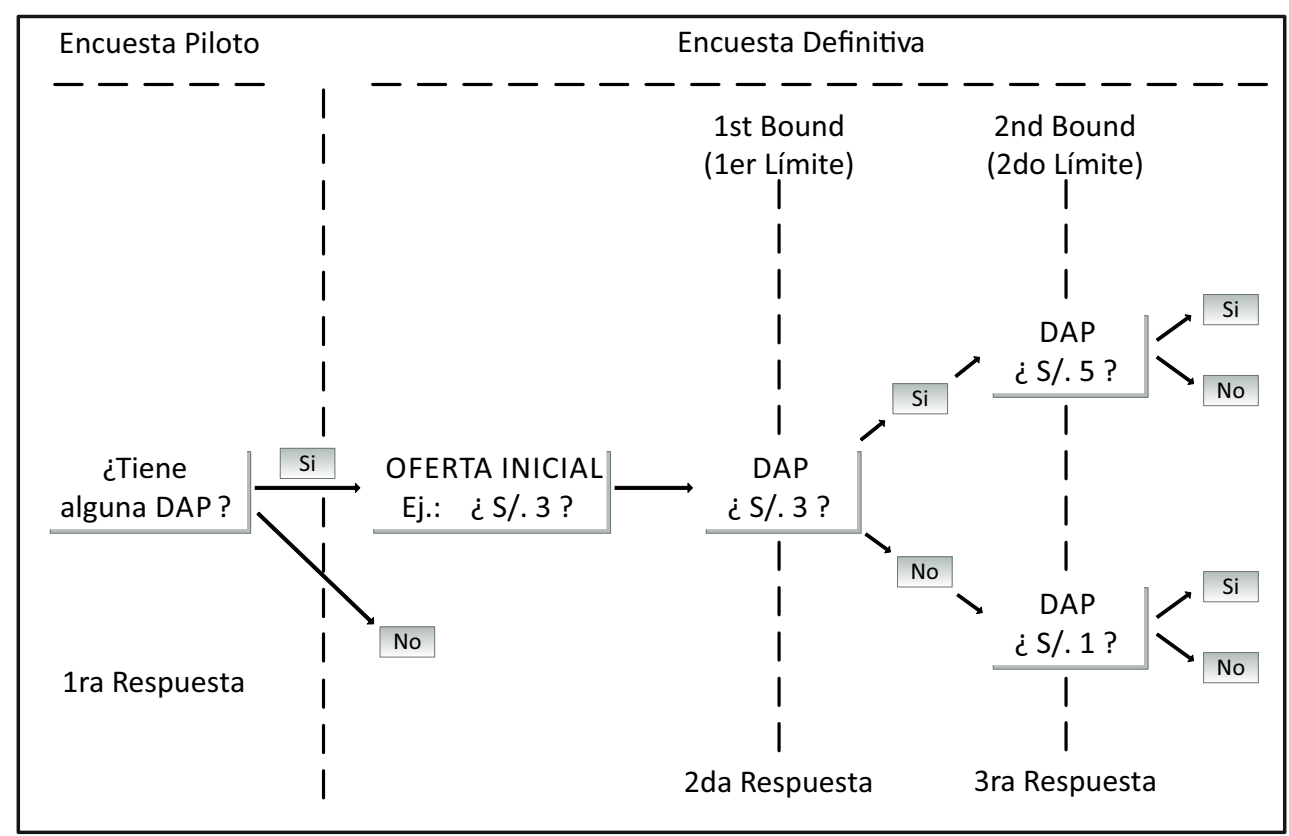

Figura 1. Estructura del formato Double Bounded utilizado. 


\section{Relación Agua - Vegetación}

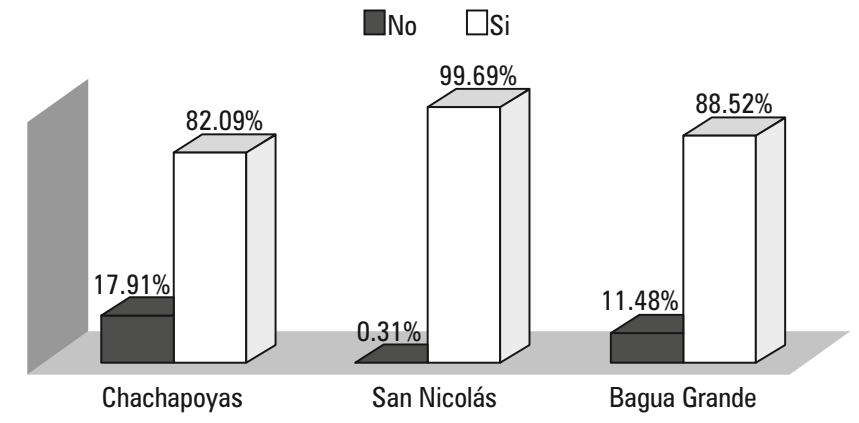

\section{Percepción sobre la Cantidad de Agua}
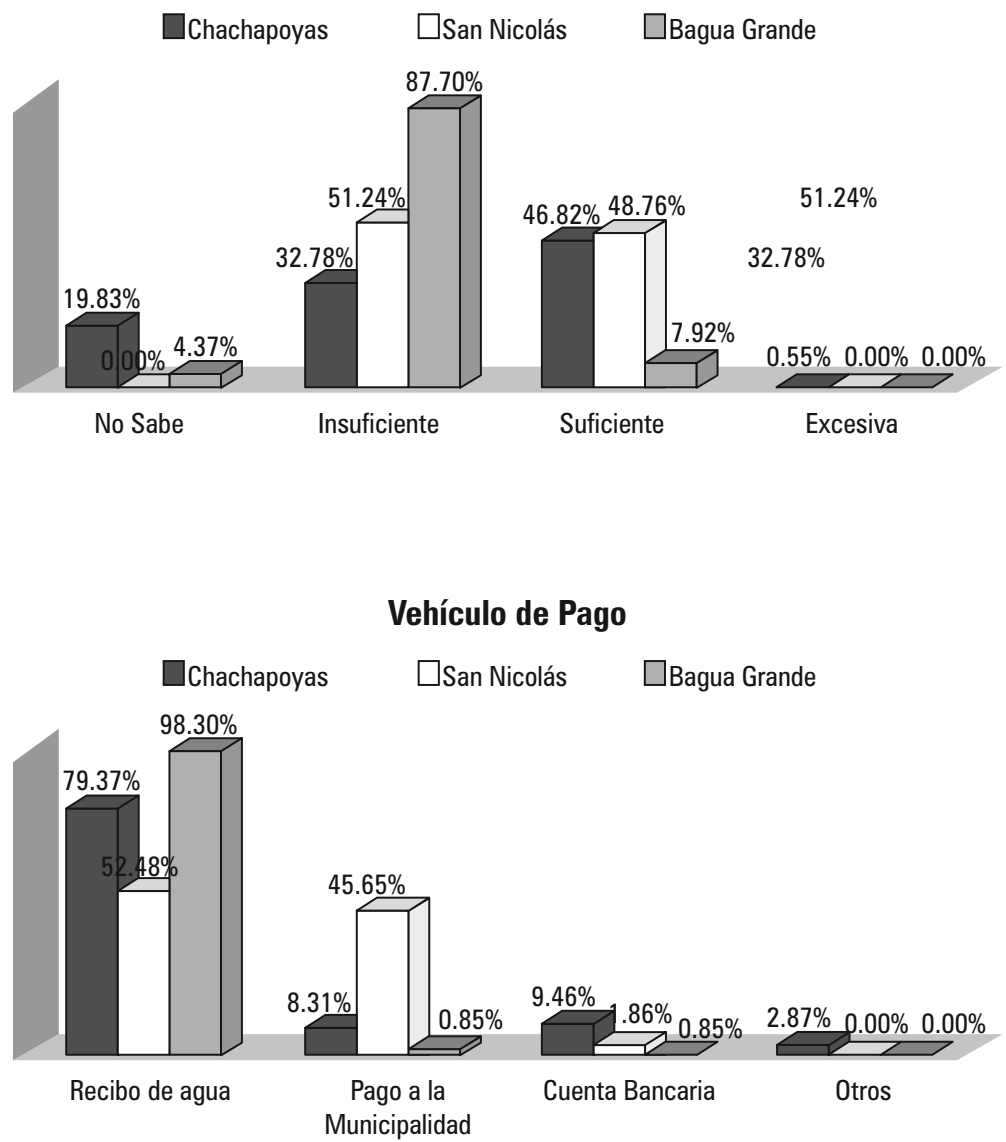

Figura 2. Estadística descriptiva en las ciudades Chachapoyas, San Nicolás y Bagua Grande. 


\section{INSTITUTO DE INVESTIGACIONES DE LA

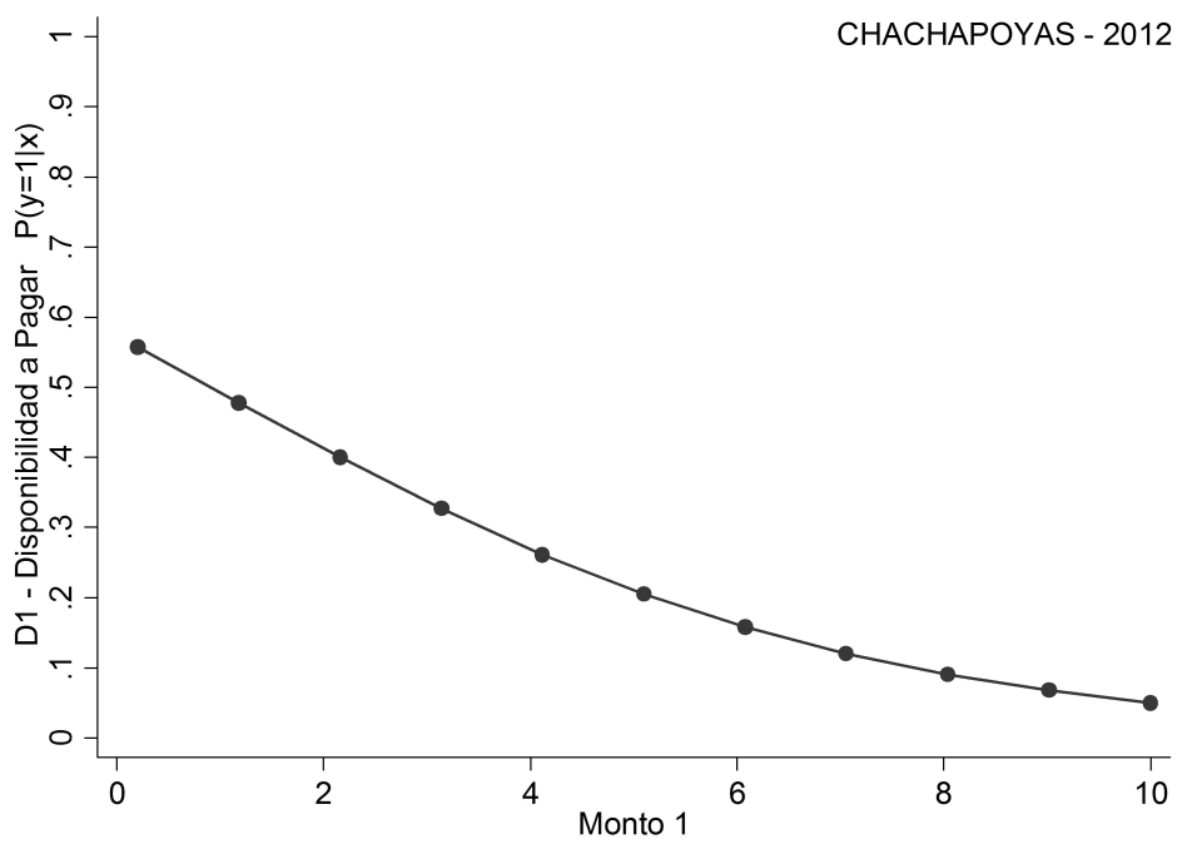

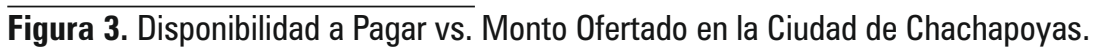

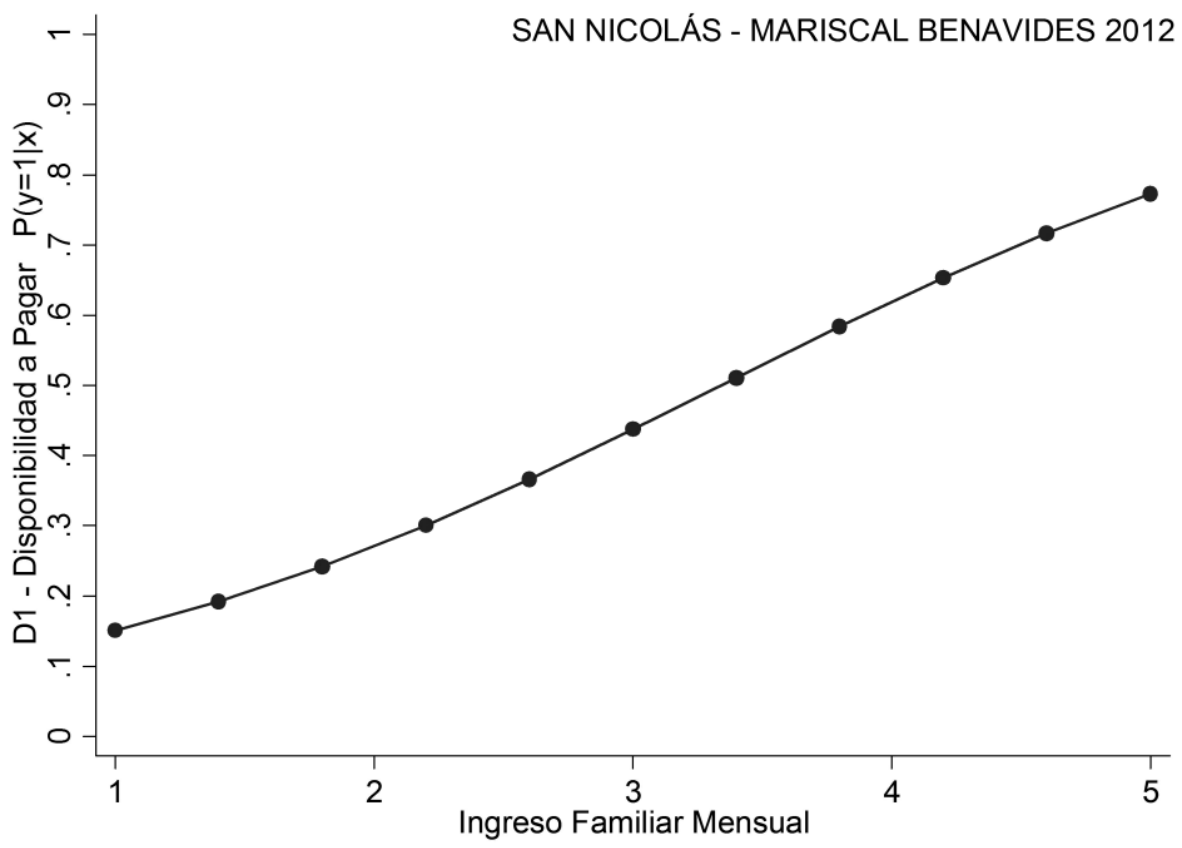

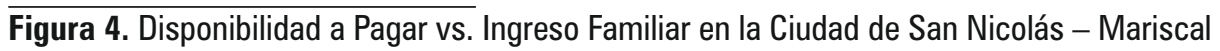
Benavides. 


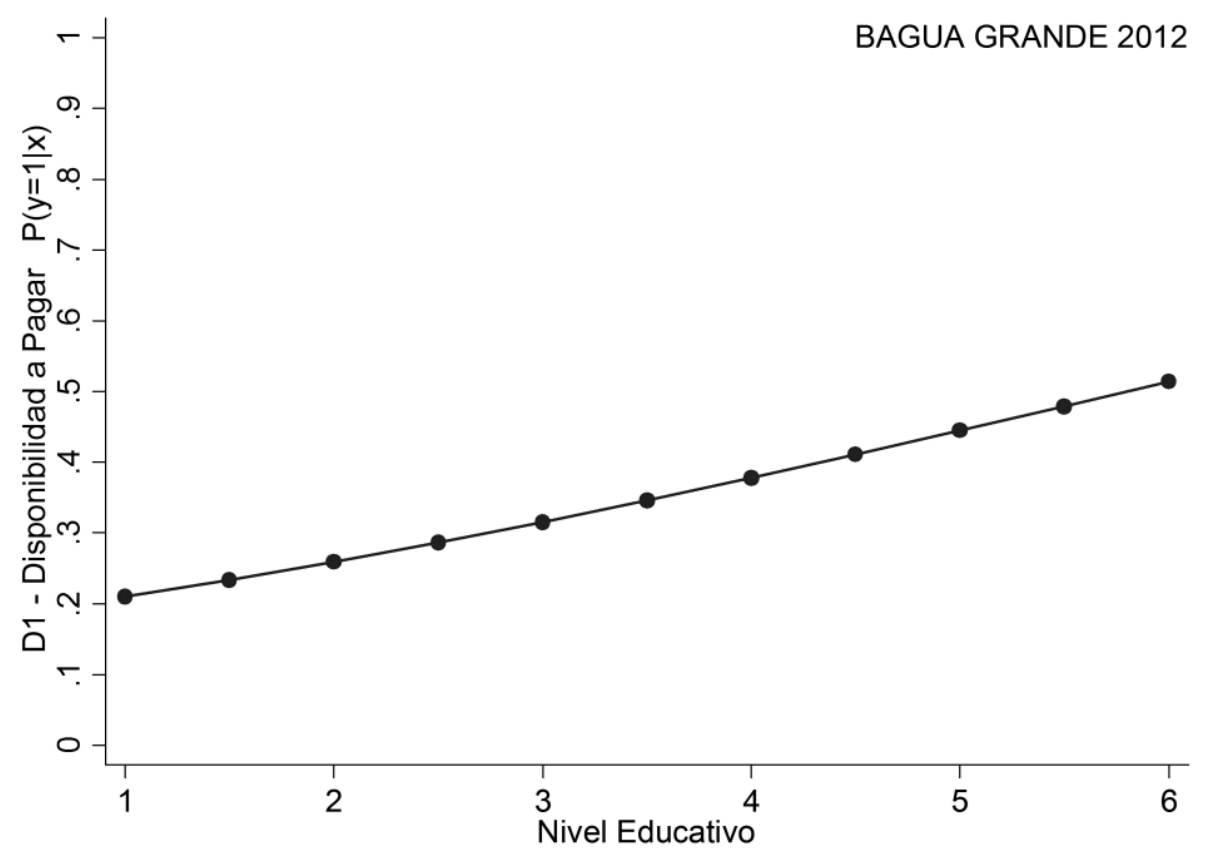

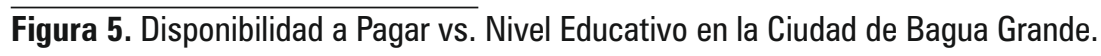

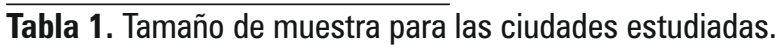

\begin{tabular}{lcc}
\hline \multicolumn{1}{c}{ Ciudad } & Hogares (N ${ }^{\circ}$ de familias) & Muestra \\
\hline Chachapoyas & 6508 & 363 \\
San Nicolás & 1791 & 317 \\
Bagua Grande & 7613 & 366 \\
\hline
\end{tabular}

Elaboración propia, con datos de INEI (2007). 
Tabla 2. Frecuencias de respuestas del formato Double Bounded

\begin{tabular}{cccccccc}
\hline \multirow{2}{*}{ Ciudad } & \multicolumn{2}{c}{ Chachapoyas } & \multicolumn{2}{c}{ San Nicolás } & \multicolumn{2}{c}{ Bagua Grande } \\
\cline { 2 - 7 } & Frecuencia & $\%$ & Frecuencia & $\%$ & Frecuencia & $\%$ \\
\hline $\mathrm{Si}-\mathrm{Si}$ & 67 & 18.46 & 41 & 12.73 & 19 & 5.19 \\
$\mathrm{Si}-\mathrm{No}$ & 64 & 17.63 & 41 & 12.73 & 101 & 27.6 \\
$\mathrm{No}-\mathrm{Si}$ & 55 & 15.15 & 54 & 16.77 & 55 & 15.03 \\
$\mathrm{No}-\mathrm{No}$ & 177 & 48.76 & 186 & 57.76 & 191 & 52.19 \\
\hline TOTAL & 363 & 100 & 322 & 100 & 366 & 100 \\
\hline
\end{tabular}

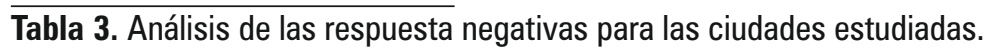

\section{Decisión \\ Decisión}

No - No (A)

Disponibilidad a asignar parte del recibo a conservación (B)

Rechazo Total (A-B)\%

Negación a cooperar $(\mathrm{DAP}=0)$

Chachapoyas

San Nicolás Bagua Grande

\begin{tabular}{ccc}
177 & 186 & 191 \\
65 & 140 & 117 \\
112 & 461 & 74 \\
$\mathbf{3 0 . 8 5}$ & $\mathbf{4 . 2 9}$ & $\mathbf{2 0 . 2 2}$ \\
\hline
\end{tabular}

Tabla 4. Estadísticos Regresión Logit formato Double Bound.

\begin{tabular}{ccccccccc}
\hline \multicolumn{3}{c}{ Chachapoyas } & \multicolumn{3}{c}{ San Nicolás } & \multicolumn{3}{c}{ Bagua Grande } \\
\hline & Variables & \multicolumn{3}{c}{ Variables } & \multicolumn{3}{c}{ Variables } \\
\hline DAP & Coef. & P $[|\mathrm{Z}|>$ z $]$ & DAP & Coef. & P $[|Z|>$ z $]$ & DAP & Coef. & P $[|\mathbf{Z}|>$ z $]$ \\
\hline M & -1.15887 & 0.0000 & M & -1.18384 & 0.0000 & M & -1.92923 & 0.0000 \\
ING & 0.892579 & 0.0000 & ING & 0.924289 & 0.0000 & ING & 0.662006 & 0.0000 \\
EDU & 0.265075 & 0.0510 & EDU & 0.064927 & 0.6324 & EDU & 0.393965 & 0.0007 \\
REL & 0.749046 & 0.0190 & CS & 0.743116 & 0.0000 & CA & 0.256594 & 0.0480 \\
Cons & -3.41854 & 0.0000 & Cons & -3.21272 & 0.0000 & Cons & -2.05887 & 0.0001 \\
\hline
\end{tabular}


Tabla 5. Resultados de la estimación de la DAP

\begin{tabular}{lccc}
\hline \multirow{2}{*}{ Indicador } & Chachapoyas & San Nicolás & Bagua Grande \\
\cline { 2 - 4 } & & $\mathbf{2 . 9 7}$ & $\mathbf{1 . 9 5}$ \\
\hline DAP (S/mes) & 15.59 & 3.45 & 2.40 \\
VAG (m3/mes) & 0.14 & 0.86 & 0.81 \\
\hline DAP (S/m3/mes) & & \\
Leyenda: & \\
DAP: Disposición a Pagar \\
VAG: Volumen promedio de agua que consumen una familia.
\end{tabular}

\section{DISCUSIÓN}

Tal como señala Hanemann et al. (1991), la DAP está en función del ingreso del entrevistado y mantiene una relación directa con el mismo.

Tudela (2012), indica que el hecho de tener un nivel de educación (EDU) cada vez mayor, aumenta la probabilidad de responder positivamente a la pregunta de disponibilidad a pagar por la implementación de políticas de gestión, esto se corrobora en el presente trabajo, sin embargo, para la ciudad de San Nicolás esta variable no resulta significativa en el modelo al $95 \%$ de confianza y para efectos de comparación se mantiene en el modelo.

Los valores capturados de la disposición a pagar presentan una relación inversa a la incidencia de la pobreza monetaria de las mismas tal que: San Nicolás, Chachapoyas y Bagua Grande presentan montos DAP de S/. 2.97, S/. 2.24 y S/. 1.95 respectivamente; en igual orden según INEI (2007), la incidencia de pobreza monetaria se encontraba en
$29.5 \% ; 33.8 \%$ y $47.7 \%$. se hace evidente la relación inversa de los montos DAP con la pobreza.

\section{CONCLUSIONES}

- La provisión de agua en las ciudades de Chachapoyas, San Nicolás y Bagua Grande, está generando mucha preocupación, por su calidad, cantidad y sostenibilidad de la explotación del servicio.

- La disposición a pagar en las ciudades de Chachapoyas, San Nicolás y Bagua Grande son $2.24,2.97$ y 1.95 nuevos soles/mes adicionales respectivamente, lo cual implica un $0.14,0.86 \mathrm{y}$ 0.81 nuevos soles $/ \mathrm{m}^{3} / \mathrm{mes}$ respectivamente.

- Modelos paramétricos tipo logit explican la decisión de disposición a pagar para implementar futuros pagos por servicios ambientales a través de: monto hipotético a pagar (M), ingreso familiar (ING), educación del encuestado (EDU) para las tres ciudades y adicionalmente, conocimiento de la relación 
entre agua y vegetación (REL), calidad del servicio (CS) y calidad del agua (CA) para las ciudades de Chachapoyas, San Nicolás y Bagua Grande respectivamente.

- La probabilidad de que un individuo responda afirmativamente ante un monto propuesto (M) es inversamente proporcional al mismo monto (M) y presenta una relación directa ante las variables ingreso familiar (ING), educación (EDU), conocimiento de la relación agua vegetación (REL), calidad del servicio de abastecimiento (CS) y calidad del agua abastecida (CA).

- El potencial recaudado anual a partir de las DAP calculadas en un eventual esquema de PSA, es de S/. 155 608. 32; S/. 55 919. 16 y S/. 192 699. 00 para Chachapoyas, San Nicolás y Bagua Grande respectivamente.

- La proporción de negación a pagar por la conservación presenta una relación inversa a problemas que presenta el servicio (cantidad y calidad del agua).

- En caso de la implementación de esquemas de PSA, los montos de la disposición de pago DAP, son de 2.24, 2.97 y 1.95 nuevos soles/mes adicionales por conexión para las ciudades de Chachapoyas, San Nicolás y Bagua Grande respectivamente, siendo el vehículo de pago más adecuado el recibo de pago por consumo de agua.

\section{AGRADECIMIENTOS}

Un especial agradecimiento a Dn. José Manuel Arellanos Briceño, por sus excelentes comentarios, consejos y sugerencias para el desarrollo de la presente investigación.

\section{BIBLIOGRAFÍA CITADA}

Ardila, S. 1993. Guía Para la Utilización de Modelos Econométricos en Aplicaciones del Método de Valoración Contingente. Documento de trabajo ENP101. Banco Interamericano de Desarrollo, Washigton, D.C. 26pp.

Arrow, K.J. 1974. "General Economic Equilibrium: Purpose, Analytic Techniques, Collective Choice". American Economic Review, American Economic Association, 64(3): 253272.

Arrow, K.; Solow, R.; Portney, P.R.; Leamer, E.E.; Schuman, R.R.H. 1993. "Report of the NOAA Panel on Contingent Valuation", Federal Register 58, 4601-4614.

Asociación Peruana para la Conservación de la Naturaleza (APECO). 2009. Propuesta de sistema de conservación regional. SICRE Amazonas. Perú. 91pp.
Azqueta, D. 2007. Introducción a la economía ambiental. Segunda edición. Editorial McGrawHill. Madrid, España. 498pp.

Habb, T.C.; McConell, K.E. 2002. Valuing environmental and natural resources: the econometric of non-market valuation. new horizons in environmental economics. Cheltenham, UK and Northampon, MA: Edward Elgar. 326pp.

Hanemann, W.M. 1984. Welfare evaluations in c o n t i n e n t valuat i o $n$ experiments with discrete responses. American $J$ o $u$ r $n$ a $l$ Agricultural Economics 66(1): 332-341

Hanemann, W.M.; Loomis, J.; Kanninen, B.1991. "Statistical Efficiency of Double Bounded Dichotomous Choice Contingent Valuation." A merican Journal of Agricultural Economics 73(4): 1255-63

Instituto Nacional de Estadística e Informática (INEI). 2007. Censo de población y vivienda. Lima, Perú. 472pp.

Instituto de Investigaciones de la Amazonía Peruana (IIAP). 2012. Zonificación Ecológica y Económica del Departamento de Amazonas (ZEE). Versión Resumida. Instituto de Investigaciones de la Amazonía Peruana IIAP y Gobierno Regional Amazonas. Lima, Perú. 52pp

Krutilla, J. 1967. "Conservation reconsidered", en American Economic Review, 57(4).

Lopez-Feldman, A. 2012. "Introduction to contingent valuation using Stata". MPRA Paper 41018, University Library of Munich, Germany. $17 \mathrm{pp}$.

Loyola, R. 2007.Valoración del Servicio Ambiental de Provisión de Agua con Base en la Reserva Nacional Salinas y Aguada Blanca - Cuenca del Río Chili. PROFONANPE, Lima, 228pp.

Ministerio del Ambiente. 2010. Compensación por servicios ecosistémicos: principios básicos de los acuerdos de conservación de servicios ecosistémicos. Las microcuencas Mishiquiyacu, Rumiyacu y Almendra de San Martín, Perú. Primera edición. Lima, Perú. 56pp.

Ministerio de Agricultura. 2009. Proyecto Especial Jaén San Ignacio Bagua (PEJSB). República del Perú. 84pp.

Pagiola, S.; Arcenas, A.; Platais, G. 2005. Can payments for environmental services help reduce poverty? An exploration of the issues and evidence to date from Latin America. World Development 33 (2), 237-253

Tudela, J. 2012. Valoración Económica y Diseño de Políticas para la Gestión Ambiental de la Reserva Nacional del Titicaca. Economía y Sociedad 80, CIES. 14pp 
